\title{
Estudios de bioequivalencia
}

\author{
Bioequivalence studies
}

Todo nuevo fármaco, sea este original (innovador) o genérico, requiere para su incorporación al arsenal terapéutico de un país, de la autorización por organismos reguladores que garanticen la seguridad y eficacia del producto. Dichos organismos exigen normalmente que las compañías farmacéuticas presenten toda la información relativa a propiedades físico-químicas, estabilidad y características farmacocinéticas de la molécula, así como los resultados de los ensayos clínicos que certifiquen la utilidad del producto innovador. Del punto de vista farmacocinético se construyen curvas que relacionan la concentración plasmática del fármaco estudiado versus tiempo, a partir de la cual se pueden determinar la concentración plasmática máxima alcanzada (Cmáx), el tiempo en alcanzar dicha concentración (Tmáx), vida media (T1/2) y el área bajo la curva (ABC), entre otros. Al relacionar las curvas obtenidas luego de administración endovenosa con las obtenidas después de la administración oral del fármaco, se puede deducir su biodisponibilidad, esto es, la estimación de la fracción absorbida del medicamento de uso oral ${ }^{1}$.

Con respecto a los compuestos genéricos, las regulaciones son más variables entre los países, exigiéndose en la mayoría sólo datos relativos a estudios de estabilidad, calidad, pureza y ensayos microbiológicos ${ }^{2}$. En EUA la Food and Drug Administration (FDA), solicita además estudios de bioequivalencia de los productos. En dichos ensayos, se recluta a sujetos sanos que reciben el fármaco original y posteriormente el compuesto genérico en estudio. Se determinan los niveles plasmáticos del fármaco y se construyen curvas en función del tiempo. Se compara entonces a los sujetos con sí mismos, en términos de Cmáx, Tmáx, T1/2 y ABC para establecer si existe o no equivalencia entre las formulaciones estudiadas. Un requisito esencial de estos estudios, es que los métodos bioanalíticos utilizados sean precisos, sensibles y reproducibles. No se recomienda efectuar estudios clínicos comparativos para demostrar bioequivalencia, por ser poco sensibles ${ }^{l}$.

En nuestro país no existe la norma de exigir a las compañías la presentación de estudios de bioequivalencia para la aprobación de fármacos genéricos, pero creemos que esta práctica sería altamente recomendable para asegurar la equivalencia de los productos que utilizamos diariamente.

En este número de la revista se presentan dos estudios de bioequivalencia de claritromicina que comparan al producto original con una nueva formulación genérica ${ }^{3,4}$. En un caso se trata de una suspensión pediátrica y en el otro, de una presentación de liberación modificada. Ambos estudios cumplen con los requisitos metodológicos de la FDA y demuestran que las dos presentaciones son bioequivalentes. A este respecto, es importante señalar, que la metodología empleada en los estudios de bioequivalencia se encuentra claramente definida y lleva en aplicación varias décadas en sus aspectos esenciales. En lo personal creo que estos estudios son de gran utilidad y deberían ser la norma para todo fármaco genérico. Surge entonces la pregunta de si este tipo de estudios corresponde realmente a una contribución científica original o sólo se trata de protocolos estandarizados que cumplen un objetivo muy específico. Personalmente me inclino por esta última opción y sin desmerecer la calidad y rigurosidad del equipo investigador, estimo que este tipo de trabajos no debiera ser publicado en revistas científicas como la Revista Chilena de Infectología sino que conformar parte del dossier de antecedentes que avalen la buena calidad de un producto genérico.

Carlos Pérez C. Departamento de Medicina 


\section{Bibliografía}

1.- Guidance for Industry. Bioavailability and Bioequivalence studies for orally administered drug products-General considerations. U.S. Department of Health and Human Services. Food and Drug Administration. Center for Drug Evaluation and Research (CDER). July 2002. www.fda.gov/cder/ guidance/4964dft.pdf

2.- Instructivo SRS. Registro nacional de productos far- macéuticos similares. Instituto de Salud Pública. www.ispch.cl/ctrl/index.html

3.- Gaete L, Schatloff O, Bello M P et al. Bioequivalencia entre dos formulaciones de claritromicina en suspensión pediátrica existentes en el mercado chileno. Rev Chil Infect 2003; 20 (3): 178-83.

4.- Gaete L, Schatloff $\mathrm{O}$, Anziani $\mathrm{F}$ et al. Bioequivalencia entre dos formulaciones de claritromicina comprimidos de liberación modificada existentes en el mercado chileno. Rev Chil Infect 2003; 20 (3): 171-7. 